\title{
38 Años de vigilancia epidemiológica de labio leporino y paladar hendido en la maternidad del Hospital Clínico de la Universidad de Chile
}

\author{
JULIO NAZER H. ${ }^{1}$, MARÍA CONSTANZA RAMÍREZ R. ${ }^{a}$, \\ LUCÍA CIFUENTES O. ${ }^{2}$
}

\section{Evolution of prevalence rates of orofacial clefts in a maternity of a Chilean clinical hospital}

Background: Orofacial clefts are common and have a great medical and social importance. The Latin American Study of Congenital Malformations (ECLAMC), has maintained an epidemiological surveillance of congenital malformations since 1969, allowing the evaluation of trends in the prevalence of malformations. Aim: To evaluate the evolution curve of prevalence rates of orofacial clefts from 1971 to 2008. Material and Methods: All cases of orofacial clefts, occurring in newborns from the maternity of a university hospital from January 2000 to December 2008, were recorded as part of the ECLAMC. Historical information about the rates of the malformation between 1971 and 1999, was obtained from previous manuscripts of the authors. Results: In the study period, 15,635 children were born and 46 had cleft lip-palate (3\%o). This rate is significantly higher than those observed previously, that fluctuated between 1.5 and $1.7 \%$. The prevalence rates of cleft lip remained stable from 1971 to 1999 and suffered a brisk and significant rise in the period 2000-2008 When the period is analyzed year by year, the increase in rates is observed in the last two years. The rates of cleft palate suffered a slight non significant rise until 2000. Conclusions: The increased rates of cleft lip palate observed in the last two years of the observation period may be a random result and should be monitored in the future.

(Rev Med Chile 2010; 138: 567-572).

Key words: Cleft lip; Congenital Abnormalities; Genetics.

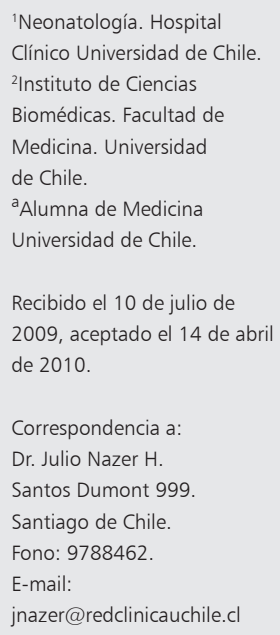

$\mathrm{E}$ 1 ECLAMC, (Estudio Colaborativo Latino Americano de Malformaciones Congénitas), al igual que el International Clearinghouse for Birth Defects Monitoring Systems (ICHBDMS) incluyen en el diagnóstico "labio leporino", a la fisura del labio y a la fisura labio-palatina. Por otra parte, "paladar hendido" corresponde a la fisura del paladar sin fisura del labio.

No se conoce completamente cual es su etiología. Según algunos autores, por ser una patología de la línea media, podría tener un origen parecido a los defectos de cierre del tubo neural, meningocele, mielomeningocele, encefalocele, etc ${ }^{1}$. Se ha sugerido que algunos factores ambientales como el tabaquismo ${ }^{2}$, el alcoholismo materno, así como deficiencias nutricionales maternas ${ }^{3}$, especialmente vitamina B6 y folatos, podrían estar involucrados en la etiología de estas anomalías ${ }^{4,5}$, hechos demostrados experimentalmente en ratas ${ }^{4}$. El déficit de ácido fólico podría ser un factor de riesgo $^{6-8}$. Otros autores lo asocian a otros factores como factores ambientales, uso de anticonvulsionantes, pesticidas, tabaco ${ }^{9-11}$. Por último, también se le ha asociado a factores genéticos ${ }^{12,13}$. 
Las frecuencias que se dan para estas patologías son variables, desde 1 en 500 nacimientos en poblaciones asiáticas, a 1 en 2.500 en africanos y 1 en 1.000 entre caucásicos, hispánicos y latinos ${ }^{14}$.

En Chile, los hospitales participantes del ECLAMC, en el período 1982-2001, entre 411.819 nacimientos daban una prevalencia de fisuras orales de 1,6 por mil. De ellos, 1,2 por mil fueron labio leporino (LL) con o sin fisura palatina y 0,5 por mil paladar hendido ${ }^{15}$.

Un hecho conocido es que LL es más frecuente en varones y $\mathrm{PH}$ en mujeres y se compromete más el lado izquierdo. El PH se observa con mayor frecuencia en formas sindrómicas y LL en patologías no sindrómicas ${ }^{16}$.

El término "no sindrómico" se refiere a individuos afectados que no presentan otras anomalías estructurales, ni corresponden a exposiciones maternas a teratógenos ambientales conocidos. Este grupo corresponde a $70 \%$ de los casos. El $30 \%$ restante están constituídos por anomalías cromosómicas y síndromes reconocibles producidos por teratógenos.

La mayoría de los casos son de etiología multifactorial, en la que intervienen factores ambientales y factores genéticos. La mitad son autonómico recesivos, $40 \%$ autosómico-dominantes y $10 \%$ ligados al sexo.

\section{Objetivos}

Estudiar la evolución de las tasas de prevalencia al nacimiento de labio leporino y paladar hendido a lo largo del tiempo, desde 1971 hasta 2008.

Comparar las prevalencias al nacimiento de las fisuras orales durante varios períodos desde enero de 1971 a diciembre del 2008 en la maternidad del Hospital Clínico de la Universidad de Chile (HCUCH). Determinar la influencia que pudo tener la implementación de la harina con ácido fólico sobre dichas frecuencias.

Se estudiará algunos antecedentes demográficos de estos niños, como peso de nacimiento, edad gestacional, catalogación, edad materna, ubicación de la fisura, malformaciones asociadas y se las comparará con los recién nacidos controles.

\section{Pacientes y Método}

La Maternidad del Hospital Clínico de la Universidad de Chile (HCUCH) es miembro del
ECLAMC desde 1969 y lleva un registro de todos los recién nacidos ( $\mathrm{RN}$ ), vivos y mortinatos, que presentan una o más anomalías congénitas. Se toma como control sano al RN del mismo sexo, sin malformaciones que nace a continuación del recién nacido malformado.

Estudio caso-control en el que se incluyeron todos los nacimientos ocurridos en la Maternidad del Hospital Clínico de la Universidad de Chile en el período comprendido entre el 1 de Enero de 2001 y el 31 de Diciembre de 2008. Ya hemos publicado las frecuencias obtenidas en períodos anteriores en la maternidad del HCUCH ${ }^{17,18}$.

Estadística: Prueba de chi cuadrado para comparar prevalencias entre los distintos períodos analizados y para contrastar las variables cualitativas, en tanto que los promedios de las variables cuantitativas (peso de nacimiento, edad materna, edad gestacional) se compararon con prueba t de Student entre casos y controles.

\section{Resultados}

En el período en estudio, 2000-2008, ocurrieron 15.635 nacimientos, de los que 15.506 fueron NV y 129 mortinatos (NM), por lo que mortinatalidad fue de $0,8 \%$. En el total de nacimientos se encontró $1.376 \mathrm{RN}$ portadores de una o más malformaciones congénitas, lo que representa una prevalencia al nacimiento de $8,9 \% ; 1.342$ eran NV $(8,6 \%)$ y 34 NM $(26,4 \% 34 / 129)$.

En esta muestra había $46 \mathrm{RN}$ (24 varones $(52,2 \%)$ y 20 mujeres $(43,5 \%)$ y 2 con sexo ambiguo $(2,3 \%)$ que presentaban fisura oral, lo que da una frecuencia de $3,0 \%$. Treinta y cinco $(2,3 \%$ o $)$ con labio leporino y $11(0,7 \%$ o) con paladar hendido. Esto constituye un aumento significativo de la prevalencia al nacimiento observada anteriormente $(\mathrm{p}=0,00087)$.

La ubicación de la fisura, a izquierda, derecha, bilateral o central, no tuvo diferencias importantes, ya que se distribuyeron en 11 casos en el lado izquierdo, 11 a derecha, 11 bilateral y 13 centrales, siendo la central más frecuente en las formas sindrómicas. Cuarenta (87\%) eran NV y 6 (13\%) NM. Veinte $(43,5 \%)$ eran formas aisladas y 26 asociadas $(56,5 \%)$.

Otras malformaciones asociadas se presentan en la Tabla 1. 
Vigilancia epidemiológica de labio leporino y paladar hendido en el HCUCH - J. Nazer H. et al

Catorce niños fueron PEG (30,4\%), 26 (56,5\%) fueron AEG y 6 (13\%) eran GEG, distribución diferente a la que se observa en $\mathrm{RN}$ sanos $(10 \%$, $80 \%$ y $10 \%$ respectivamente).

El promedio de peso de nacimiento fue de 2.674,67 gramos, con un rango de 345 g (PEG de 24 semanas nacido vivo) y 4.930 g, promedio

Tabla 1. Malformaciones asociadas con LL y PH

\begin{tabular}{|lc|}
\hline Diagnósticos & n de casos \\
\hline SNC & 7 \\
\hline MF Esqueléticas & 6 \\
\hline Trisomía 13 & 4 \\
\hline Trisomía 18 & 3 \\
\hline Trisomía 21 & 1 \\
Cardíacas & 4 \\
\hline Renales & 3 \\
\hline Otras & 7 \\
\hline
\end{tabular}

significativamente inferior al de recién nacidos controles $(3.321, \mathrm{p}<0,0001)$.

El promedio de edad gestacional fue de 35,89 semanas, con un rango de 24 y 40 semanas.

El promedio de edad materna fue de 31,39 años, con un rango de 16 y 42 años, promedio superior al encontrado en recién nacidos controles $(29,4$ años, $\mathrm{p}<0,032)$.

En 18 pacientes $(39,1 \%)$, había antecedentes de otro malformado en la familia, de los cuales 5 casos $(10,9 \%)$, había un familiar con fisura labiopalatina, lo que está sugiriendo que hay algún factor genético comprometido.

Al estudiar la evolución en el tiempo de estas tasas (Tabla 2 y Figura 1), podemos observar que el aumento de ellas en el período 1971 hasta 2008 es diferente en LL y PH. En efecto, las tasas de LL se mantienen prácticamente estables en el tiempo hasta 1999. Sin embargo, en el período 2000-2008 presentan un aumento brusco y significativo ( $\mathrm{p}=0,00087)$ respecto a los períodos anteriores, debido particularmente al aumento de la tasa en este último período, no así PH cuya

Tabla 2. Evolución de la prevalencia de labio leporino y paladar hendido en la maternidad del Hospital Clínico de la Universidad de Chile. Período 1971- 2008. Tasas por mil

\begin{tabular}{|lcccccc|}
\hline Diagnóstico & \multicolumn{5}{c|}{ Períodos } \\
& $\mathbf{1 9 7 1 - 1 9 7 7}$ & $\mathbf{1 9 7 2 - 1 9 8 4}$ & $\mathbf{1 9 8 2 - 1 9 8 8}$ & $\mathbf{1 9 8 9 - 1 9 9 4}$ & $\mathbf{1 9 9 5 - 1 9 9 9}$ & $\mathbf{2 0 0 0 - 2 0 0 8}$ \\
\hline Labio leporino & 1,2 & 1,3 & 1,2 & 1,1 & 1,2 & 2,3 \\
Paladar hendido & 0,3 & 0,3 & 0,5 & 0,5 & 0,5 & 0,7 \\
\hline Total & 1,5 & 1,6 & 1,7 & 1,6 & 1,7 & 3,0 \\
\hline
\end{tabular}

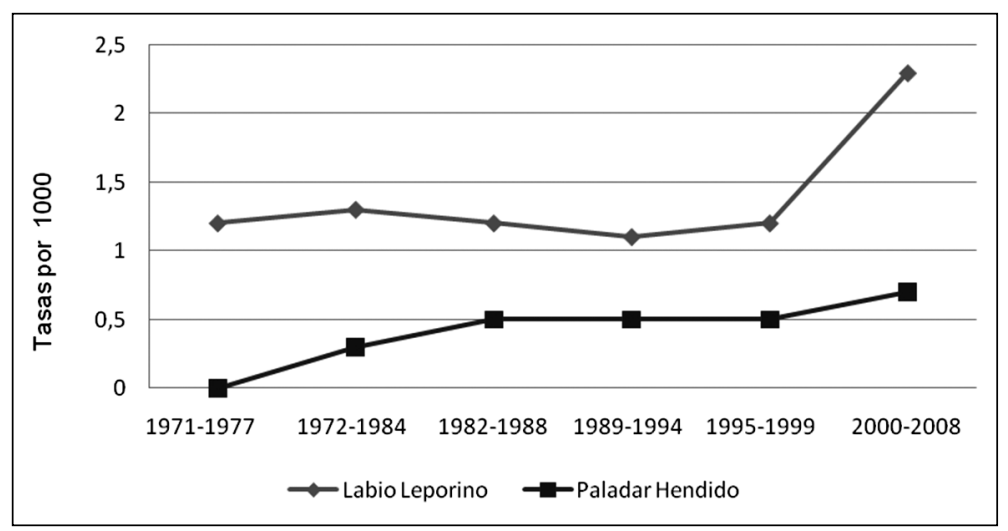

Figura 1. Evolución de la prevalencia de labio leporino y paladar hendido. 
Vigilancia epidemiológica de labio leporino y paladar hendido en el HCUCH - J. Nazer H. et al

Tabla 3. Evolución de las tasas de prevalencia al nacimiento de labio leporino, paladar hendido y total de fisuras labio palatinas. Período 2000-2008

\begin{tabular}{|lccccccccc|}
\hline & \multicolumn{10}{c|}{ Tasas por $\mathbf{1 . 0 0 0}$} \\
Diagnóstico & $\mathbf{2 0 0 0}$ & $\mathbf{2 0 0 1}$ & $\mathbf{2 0 0 2}$ & $\mathbf{2 0 0 3}$ & $\mathbf{2 0 0 4}$ & $\mathbf{2 0 0 5}$ & $\mathbf{2 0 0 6}$ & $\mathbf{2 0 0 7}$ & $\mathbf{2 0 0 8}$ \\
Labio leporino & 0,9 & 2,3 & 2,7 & 2,5 & 1,9 & 2,0 & 1,3 & 2,5 & 4,8 \\
Paladar hendido & 0,0 & 0,9 & 0,5 & 0,0 & 0,6 & 0,6 & 0,7 & 1,9 & 0,6 \\
Total fisuras & 0,9 & 3,2 & 3,2 & 2,5 & 2,5 & 2,6 & 2,0 & 4,4 & 5,4 \\
\hline
\end{tabular}

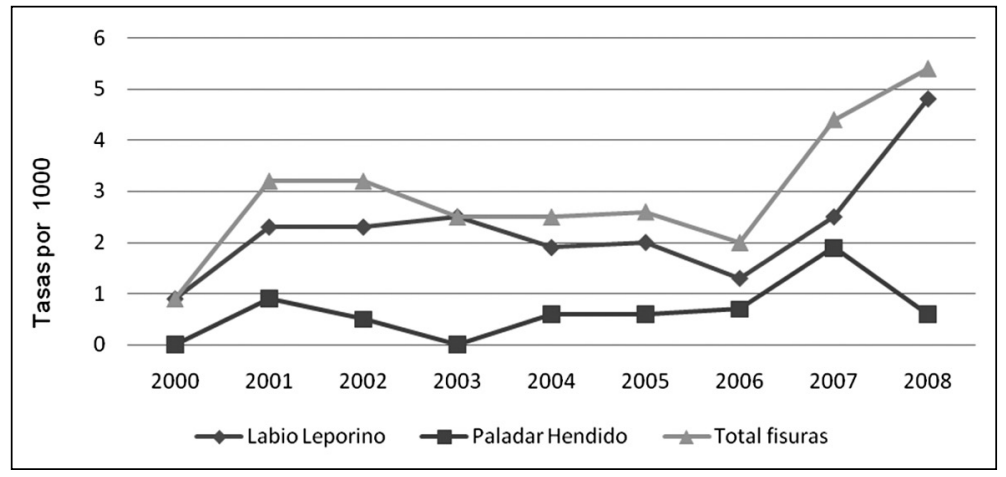

Figura 2. Evolución de la prevalencia de labio leporino, paladar hendido y total de fisuras labio palatinas.

alza ha sido leve, pero sostenida, incrementándose desde $1971(0,3 \%$ o hasta $1999(0,5 \%$ o $)$ y $0,7 \%$ el último período 2001-2008. Las tasas de fisura palatina no difieren significativamente entre los períodos analizados $(\mathrm{p}=0,11)$

Para tratar de encontrar la causa de este incremento de la tasa de labio leporino construimos una tabla con las tasas de LL, PH y total de las fisuras labio-palatinas desde el año 2000 al 2008 (Tabla 3 y Figura 2). Se puede observar que el aumento de LL ocurre a partir del año 2001, pero este incremento se acentúa en forma más rápida en los dos últimos años.

\section{Discusión}

Las tasas de fisuras labio palatinas son significativamente diferentes entre los períodos analizados $(\mathrm{p}=0,0087)$ debido particularmente al aumento de la tasa de labio leporino en el período 20012008 comparativamente con todos los anteriores. Sin embargo, el incremento más notorio se produce en los dos últimos años. Paladar hendido por el contrario mantiene sus tasas en forma estable, salvo un aumento transitorio en el año 2007.

¿Como explicar este fenómeno? ¿Qué ocurrió desde el año 2001 que hizo variar las tasas de LL que se habían mantenido estables, y no así las de PH que tenían un aumento sostenido desde 1971?.

El hecho más significativo que ocurrió ese año fue la fortificación de la harina con ácido fólico con el objetivo de disminuir la incidencia de defectos de cierre del tubo neural, hecho que fue confirmado con una disminución significativa de incidencia de espina bífida en cerca de $60 \%{ }^{17-19}$. Se pensó que otras patologías también pudieran beneficiarse con esta medida, entre ellas labio leporino. Shaw ${ }^{26}$ comunica una reducción del riesgo entre 24 y $50 \%$ para los hijos de mujeres que habían recibido multivitamínicos, que contenían ácido fólico, periconcepcionalmente. Padmaja ${ }^{21}$ encontró $48 \%$ de disminución del riesgo para labio leporino con o sin paladar hendido en los hijos de mujeres que usaron multivitamínicos en el período periconcepcional. El ECLAMC en estudios preliminares no logró demostrarlo ${ }^{20}$. En estudios nuestros tampoco lo demostramos ${ }^{21,22}$. 
Labio leporino permaneció estable. Sin embargo, PH aumentó en forma significativa $(\mathrm{p}<0,05)$ al igual que otras malformaciones como atresia de duodeno, hidro y microcefalia, hidronefrosis, riñón poliquístico, síndrome de Down, que podrían tener otras explicaciones, como mejor diagnóstico por ecografías y derivación al hospital para ser atendido en mejores condiciones. Síndrome de Down es una patología que ha venido aumentando su prevalencia al nacimiento desde hace algún tiempo ${ }^{21-23}$.

La mayoría de la literatura consultada nos muestra que habría evidencias que sugieren que la administración de ácido fólico ayudaría en la prevención de fisuras orales ${ }^{5-9,19,21,22,24}$. Por el contrario, encontramos escasa evidencia que nos muestre un aumento de las tasas provocadas por la administración de ácido fólico. Nuestros resultados no son concluyentes en este aspecto, ya que LL c/s PH sólo modifican su frecuencia después de 6 años de iniciada la fortificación de la harina con ácido fólico. El aumento brusco y significativo de los últimos dos años debe tener un origen diferente, que desconocemos.

Por tratarse de un importante problema de salud pública que repercute en lo económico, social y familiar, seguiremos vigilando el comportamiento de las tasas de fisuras orales en nuestro hospital por un tiempo más, para poder afirmar o descartar la influencia del ácido fólico como causa de sus variaciones.

\section{Referencias}

1. Czeizel AE, Timar L, Sankozi A. Dose-dependent effect of folic acid on the prevention of orofacial clefts. Pediatrics 1999; 104: e66.

2. Lammer E, Shaw GM, Iovannisei D, Van Waes J, Finnel RH. Maternal smoking and risk of orofacial clefts. Epidemiology 2004; 15: 150-6.

3. Shaw GM, Carmichael SL, Laurent C, Rasmussen SA. Maternal nutrient intakes and risk of orofacial clefts. Epidemiology 2006; 17: 285-91.

4. Shaw GM, Lamer E, Wasserman CR, O'Malley CD, Tolarova MM. Risks of orofacial clefts in children born to women using multivitamins containing folic acid periconceptionally. Lancet 1995; 346: 393-6.

5. Warkany J, Nelson RC, Schrahenbergern E. Congenital malformation induced in rats by maternal nutritional deficiency. Am J Dis Child 1943; 65: 882-94
6. Ray JG, Meier C,Vermeulen MJ, Wyatt PR, Cole D. Association between folic acid fortification and congenital orofacial clefts. J Pediatr 2003; 143: 805-7.

7. Hayes C. Werler MM, Willett WC, Mitchell AA. Casecontrol study of periconceptional folic acid supplementation and oral clefts. Am J Epidemiol 1996; 143: 1229-34.

8. Rouget F, Monfort C, Bahuau M. Periconceptional folates and the prevention of oral clefts: rol of dietary intakes in France. Rev Epidemiol Sante Publique 2005; 53: 351-60.

9. Jackson RJ. Maternal pesticide exposure from multiple sources and selected congenital anomalies. Epidemiology 1999; 10: 60-6.

10. Rull R, Ritz b, Shaw GM. Neural tube defects and maternal residential proximity to agricultural pesticide applications. Am J Epidemiology 2006; 163: 743-53.

11. Carinci F, Rullo R, Farina A, Morano D, Festa VM, Mazzarella N, et al. Nonsindromic orofacial clefts in Southern Italy: pattern analysis according to gender, maternal history of maternal smoking, folic acid intake and familial diabetes. J Craniomaxillofac Surg 2005; 33 : 91-4.

12. Bender PL. Genetics of cleft lip and palate. J Pediatr Nurs 2000; 15: 242-9.

13. Padmanabham R. Etiology, pathogenesis and prevention of neural tube defects. Congenit Anom 2006; 46: 55-67.

14. Zhu J, Ren A, Zhu H, Li S, Finnell RH, Li Z. Variable contribution of the MTHFR C677T polymorphism to non-sindromic cleft lip and palate risk in China. Am J Med Genet A 2006; 140: 551-7.

15. Nazer J. Fisuras orales. En Hübner, Ramírez, Nazer J. Malformaciones congénitas. Diagnóstico y manejo neonatal. Ed. Universitaria. 2005; 91-5.

16. Nazer J, Cifuentes L, Meza M. Incidencia de las malformaciones congénitas en 10 maternidades chilenas participantes del ECLAMC. Comparación de tres períodos. Rev Med Chile 1997; 125: 993-1001.

17. Nazer J, Aravena T, Cifuentes L. Malformaciones congénitas. Un problema emergente. Período 1995-99. Rev Med Chile 2001; 895-904.

18. Nazer J, Hübner ME, Catalán J, Cifuentes L. Incidencia de labio leporino y paladar hendido en la maternidad del Hospital Clínico de la Universidad de Chile y en las maternidades chilenas participantes del ECLAMC. Período 1991-1999. Rev Med Chile 2001; 285-93.

19. Padmaja RM, Watkins M, Moore C, Liu Y. Maternal multivitamin use and orofacial cleft in offspring. Teratology 2001; 63: 79-86.

20. Castilla EE, Orioli IM, López-Camelo J, Graca Dutra M, Nazer J. Prelimary data on changes in neural tube 
prevalence rates alter folic acid fortification in South America. Am J of Medical Genetics 2003; 123A: 1238.

21. López-Camelo J, Orioli M, Graca Dutra M, Nazer J, Rivera N, Ojeda ME. Reduction of birth prevalence rates of neural Tube defects alter folic acid fortification in Chile. Am J of Medical Genetics 2005; 135A: 120-5.

22. Nazer J, Cifuentes L, Aguila A, Juárez ME, Cid MP, Godoy ML, et al. Efecto de la fortificación de la harina con ácido fólico sobre la evolución de las tasas de prevalencia al nacimiento de malformaciones congénitas en los hospitales chilenos del ECLAMC. Rev Med Chile 2007; 135: 198-204.

23. Nazer J, Aguila A, Cifuentes L. Vigilancia epidemiológica del Síndrome de Down en el Hospital Clínico de la Universidad de Chile. Período 1972-2005. Rev Med Chile 2006; 134: 1436-

24. Chávez-Corral DV. Relation between levels of folic acid, vitamin B12 and homocysteine with neural tube defects and cleft lip". Int J Morphol 2008; 26 (4): 905-14. 